INPLASY

PROTOCOL

To cite: Cheng et al. Early physical rehabilitation vs standard care for intracerebral hemorrhage stroke: a protocol for systematic review and meta analysis. Inplasy protocol 2020110068. doi: 10.37766/inplasy2020.11.0068

Received: 16 November 2020

Published: 17 November 2020

Corresponding author:

Fang Zhang

1207Ihj@sina.com

Author Affiliation:

Department of Rehabilitation Medicine, Second Hospital of Lanzhou University

Support: Gansu National Science, No.201.

Review Stage at time of this submission: Preliminary searches.

Conflicts of interest:

The authors claimed there was no conflicts of interest.

\section{Early physical rehabilitation vs standard care for intracerebral hemorrhage stroke: a protocol for systematic review and meta analysis}

Cheng, X1; Mao, S2; Zhang, Y3; Peng, X4; Ma, R5; Bao, Y6; Li, Q7; Liu, M8; Sun, D9; Wan, B10; Wang, L'11; Zhang, F12.

Review question / Objective: This systematic review aimed to investigate whether early physical rehabilitation could have a beneficial effect for the patient with intracerebral hemorrhage stroke compared with standard rehabilitation care.

Condition being studied: The patient with intracerebral hemorrhage stroke.

Information sources: A systematic search of the literature will be conducted using the PubMed, Web of science, Cochrane library, and four Chinese databases, which including Chinese Biomedical Databases (CBM), China National Knowledge Infrastructure (CNKI), Wanfang and Chongqing VIP. No restrictions in language, publication date or publication year are applied. Additional source including WHO clinical trial registry website, clinicaltrail.gov, conference abstracts, will also be searched. Further, the references of included trails will also be checked for more potential studies.

INPLASY registration number: This protocol was registered with the International Platform of Registered Systematic Review and Meta-Analysis Protocols (INPLASY) on 17 November 2020 and was last updated on 14 December 2020 (registration number INPLASY2020110068).

\section{INTRODUCTION}

Review question / Objective: This systematic review aimed to investigate whether early physical rehabilitation could have a beneficial effect for the patient with intracerebral hemorrhage stroke compared with standard rehabilitation care.
Condition being studied: The patient with intracerebral hemorrhage stroke

\section{METHODS}

Participant or population: The patient with intracerebral hemorrhage stroke. 
Intervention: Early physical rehabilitation.

Comparator: Standard rehabilitation care or usual care.

Study designs to be included: Randomized controlled trials.

Eligibility criteria: The RCTs which investigate the rehabilitation effective of the early mobilization for the intracerebral hemorrhage compared with routine nursing or standard care will be eligible for this systematic review.

Information sources: A systematic search of the literature will be conducted using the PubMed, Web of science, Cochrane library, and four Chinese databases, which including Chinese Biomedical Databases (CBM), China National Knowledge Infrastructure (CNKI), Wanfang and Chongqing VIP. No restrictions in language, publication date or publication year are applied. Additional source including WHO clinical trial registry website, clinicaltrial.gov, conference abstracts, will also be searched. Further, the references of included trails will also be checked for more potential studies.

Main outcome(s): The primary outcomes include the mortality, the functional performance and quality of life evaluated by the Functional Independence Measure (FIM-motor), Postural Assessment Scale for Stroke Patients, and Functional Ambulation Category (FAC), Modified Barthel Index, Short Form-36 (SF-36), and other validated scales. The secondary outcomes include the length of stay, recurrent stroke, and adverse effects.

Quality assessment / Risk of bias analysis: The risk of bias (ROB) for included studies will be assessed using the Cochrane collaboration's tool by two independent reviewers form the following dominants: the assessment includes sequence generation; allocation concealment; blinding of participants, personnel, and outcome assessors; incomplete outcome data; selective outcome reporting; and other sources of bias.
Strategy of data synthesis: RevMan version 5.3 will be used to conduct all calculations related to the meta-analysis. Dichotomous data will be calculated in terms of a fixed or random effect model and expressed by the relative risk (RR) with $95 \%$ confidence interval ( $\mathrm{Cl})$. Continuous data will be presented as mean difference and $95 \% \mathrm{CI}$. The inconsistency index (12) and Chisquared will be calculated for heterogeneity detection between studies. When assessing the difference in outcome, heterogeneity involving all trials will be examined. A value of $\mathrm{P}<0.05$ will be considered statistically significant.

Subgroup analysis: The subgroup will be performed based on the several participants characters (including the various severity of stroke and different age), and therapy duration and start time of intervention, and the studies qualities according to the assessment results of risk of bias, etc.

Sensibility analysis: Sensitivity analysis will be carried out for primary outcomes by removing one study a time to investigate the robustness of the meta-analysis result.

\section{Country(ies) involved: China.}

Keywords: Rehabilitation; early physical rehabilitation; intracerebral hemorrhage stroke; systematic review.

Contributions of each author:

Author 1 - Xiaorong Cheng.

Author 2 - Shunqin Mao.

Author 3 - Yibao Zhang.

Author 4 - Xiaoyun Peng.

Author 5 - Rui Ma.

Author 6 - Yingcun Bao.

Author 7 - Qun Li.

Author 8 - Mei Liu.

Author 9 - Dengjuan Sun.

Author 10 - Bo Wan.

Author 11 - Lulu Wang.

Author 12 - Fang Zhang. 